\title{
Effective and less invasive diagnostic strategy for gastrointestinal GVHD
}

\section{다 (우우}

\begin{abstract}
Authors
Institutions

1 Division of Gastroenterology, Tohoku University Graduate School of Medicine, Sendai, Japan

2 Department of Pathology, Tohoku University Hospital, Sendai, Japan
\end{abstract}

Katsuya Endo ${ }^{1}$, Fumiyoshi Fujishima², Masatake Kuroha ${ }^{1}$, Rintaro Moroi ${ }^{1}$, Motoyuki Onodera ${ }^{1}$, Takeo Naito ${ }^{1}$, Yoshitake Kanazawa', Tomoya Kimura', Hisashi Shiga', Yoichi Kakuta', Yoshitaka Kinouchi' ${ }^{1}$, Tooru Shimosegawa ${ }^{1}$

submitted 28.3.2017

accepted after revision 31.8 .2017

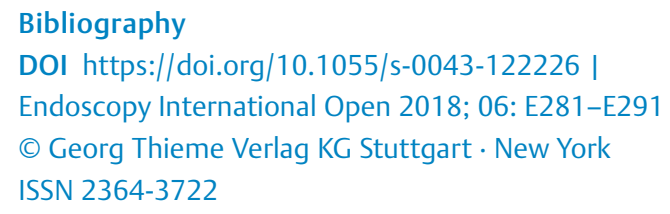

Corresponding author

Katsuya Endo, MD, PhD, Division of Gastroenterology, Tohoku University Graduate School of Medicine, 1-1 Seiryo, Aoba-ku, Sendai 980-8574, Japan

Fax: +81-22-717-7177

kendo@med.tohoku.ac.jp

\section{ABSTRACT}

Background and study aims Rectosigmoidoscopy with biopsy has been regarded to be a useful procedure to diagnose gastrointestinal graft-versus-host disease (GVHD). However, little is known about the specific colonoscopic features of gastrointestinal GVHD. In this study, we focused on the 4 unique colonoscopic findings - orange peel appearance, spotty redness, small mucosal sloughing, and diffuse mucosal defect - which are possible specific findings of gastrointestinal GVHD. We aimed to estimate the usefulness of these four unique colonoscopic findings in the rectosigmoid portion to diagnose gastrointestinal GVHD.
Patients and methods Seventy patients who were histologically diagnosed with gastrointestinal GVHD at our institute were retrospectively enrolled. Colonoscopic findings were reviewed, focusing on the four characteristic findings. The percentage of the positive cases for the characteristic findings was calculated. The final scoping portion and the number of cases showing any of the four characteristic findings in the rectosigmoid portion were also evaluated. The relationships between biopsy sites and the histological findings were also evaluated.

Results Orange peel appearance was observed in 66 cases (94.3\%). Spotty redness was observed in 45 cases (64.3\%). Small mucosal sloughing was observed in 49 cases $(70.0 \%)$. Diffuse mucosal defect was observed in six cases (8.6\%). The number of cases that were concurrently positive for one, two, and three findings were 16 (20.8\%), 20 (26.0\%), and 34 (48.6\%), respectively. Fifty-eight cases (82.9\%) were investigated up to the rectosigmoid portion, and 12 (17.1\%) were investigated beyond the sigmoid colon. All of the cases showed at least 1 of the 4 characteristics in the rectosigmoid portion. The percentage of crypt apoptosis in the biopsy specimen from orange peel appearance, spotty redness, small mucosal sloughing, and diffuse mucosal defect were $87.5 \%, 83.3 \%, 87.2 \%$, and $88.9 \%$, respectively. Conclusion Orange peel appearance, spotty redness, small mucosal sloughing, and diffuse mucosal defect are the characteristic colonoscopic findings useful for diagnosis of gastrointestinal GVHD. These findings are frequently observed in the rectosigmoid portion. The histological detection rates for crypt cell apoptosis from these findings are high. Identifying the four characteristic findings on rectosigmoidoscopy and taking biopsies from these areas could be essential for the diagnostic strategy for gastrointestinal GVHD.

\section{Introduction}

Acute graft-versus-host disease (GVHD) is a serious complication after hematopoietic stem cell transplantation (HSCT), and is a major cause of mortality. It often occurs within 100 days following HSCT and mainly involves the skin, liver, and gastroin- testinal tract. The gastrointestinal tract is the second most frequently involved organ, following the skin. Since gastrointestinal GVHD often leads to a poor outcome [1,2], making an immediate diagnosis is essential to improving the prognosis after HSCT. Watery diarrhea, nausea, vomiting, abdominal pain, and 
hematochezia are frequent symptoms of acute gastrointestinal GVHD. However, it is difficult to make an accurate diagnosis only from the clinical symptoms, because these are not specific for gastrointestinal GVHD. The gold standard for diagnosing acute gastrointestinal GVHD is the histological findings from an endoscopic mucosal biopsy.

For prompt and efficient diagnosis of gastrointestinal GVHD, the biopsy portion of the gastrointestinal tract with the highest diagnostic yield has been a topic of debate. The duodenum is the most frequently involved segment of the upper gastrointestinal tract. Therefore, a biopsy from the duodenum is important for diagnosing gastrointestinal GVHD, even if the endoscopic finding of the duodenum is normal [3]. However, in many of the patients, the upper gastrointestinal endoscopy with biopsy is not adequate for diagnosis because of the low frequency of upper gastrointestinal lesions in gastrointestinal GVHD. Many recent studies have reported that a biopsy from the lower intestinal tract has equal or superior sensitivity for the pathological diagnosis of gastrointestinal GVHD compared to a biopsy from the upper gastrointestinal tract [4-9]. Furthermore, some studies reported that a biopsy from the rectosigmoid portion, rather than from the right colon or the ileum, is sufficient for diagnosis [8, $10-12]$. Since upper GI endoscopy and total colonoscopy are more invasive than rectosigmoidoscopy, rectosigmoidoscopy with biopsy has been regarded as the more useful way to diagnose gastrointestinal GVHD in recent years. However, little is known about the specific colonoscopic features of gastrointestinal GVHD. Several studies reported that gastrointestinal GVHD shows various and non-specific colonoscopic findings and that it is difficult to identify the characteristic findings $[3,4,13,14]$. Conversely, the orange peel appearance or tortoiseshell pattern, which represents mucosal edema with a granular surface, was described as the specific finding of gastrointestinal GVHD in several reports [15-18]. In addition, spotty redness and small mucosal sloughing were also described as possible specific findings of gastrointestinal GVHD in several small case series $[17,18]$. However, the numbers of cases in these previous studies was too small to establish reliable evidence. In our clinical experiences, these characteristic findings are frequently observed in the rectosigmoid portion. In addition, we have also noticed that diffuse mucosal defect could be the characteristic finding of gastrointestinal GVHD. Although these unique colonoscopic findings could be specific features of gastrointestinal GVHD, there have been few reports that evaluated the frequency of these findings in the rectosigmoid portion. Furthermore, corresponding histological findings of these endoscopic features have not been evaluated.

In the current study, we aimed to estimate the usefulness of the four unique colonoscopic findings, orange peel appearance, spotty redness, small mucosal sloughing, and diffuse mucosal defect in the rectosigmoid portion, for diagnosing gastrointestinal GVHD.

\section{Patients and methods}

\section{Patients}

We retrospectively reviewed the colonoscopic features of patients who were diagnosed with gastrointestinal GVHD after allogenic HSCT, between January 2001 and December 2015 at Tohoku University Hospital. gastrointestinal GVHD was diagnosed based on the histological findings. Histological gastrointestinal GVHD was defined as presence of gland apoptosis, not explained by other inflammatory or infectious etiologies. Patients diagnosed with gastrointestinal GVHD complicated with Cytomegalovirus (CMV) colitis were excluded in this study.

\section{Colonoscopy procedure}

The colonoscopies were performed by 4 expert endoscopists, all of whom had more than 10 years and experience and had performed more than 5,000 colonoscopies. The colonoscopies were performed with no bowel preparations because most of the cases had watery diarrhea and were in poor general conditions. The colonoscopies were performed using Olympus CFQ240ZI, CF-H260AI, CF-H260AZI, PCF-Q260JI, and PCFQ260Al. The scope selections depended on each colonoscopist's decision. All cases were investigated by colonoscopy with white-light and chromoendoscopy with indigo carmine. In some cases, magnifying procedures were added. The determinations of performing rectosigmoidoscopy or total colonoscopy depended on the patient's general conditions which were evaluated by each colonoscopist.

\section{Methods}

Diagnostic sensitivity of the four characteristic colonoscopic findings for gastrointestinal GVHD

Colonoscopic findings of the patients with gastrointestinal GVHD were reviewed, retrospectively. The colonoscopic findings were investigated by reviewing all captured images and the endoscopy reports. Colonoscopic findings were reviewed, retrospectively. Colonoscopic findings were reviewed, focusing on the following four characteristic findings: a) orange peel appearance, b) spotty redness, c) small mucosal sloughing, and d) diffuse mucosal defect. Pictures of these findings are demonstrated in $\mathbf{F i g . 1}$. Orange peel appearance was defined as mucosal edema with a granular surface looking like "orange peel" or "tortoiseshell," which was usually made more visible on indigo carmine dye spraying ( $\triangleright$ Fig. 1a). Spotty redness was defined as redness of 1 to $2 \mathrm{~cm}$ in diameter with an unclear border ( $\triangleright$ Fig. 1b). Small mucosal sloughing was defined as small areas of superficial mucosal sloughing, with various shapes, of which the border is clear ( Fig. 1c). Diffuse mucosal defects were defined as large ulcers with irregular or geographic shapes ( $\triangleright$ Fig. 1d).

The percentage of positive cases with each of the above characteristic findings was calculated. Combinations of the 4 findings were also evaluated.

The final scoping portion and the number of positive cases for any of the 4 characteristic findings in the rectosigmoid portion were also evaluated. 


\section{Definitions of the four characteristic findings}

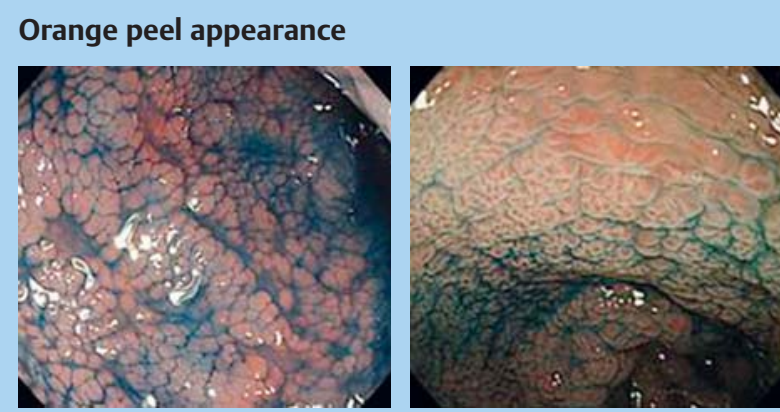

- Mucosal edema looking like „Orange peel“ or „Tortoiseshell“

- Highlighted with indigo carmine dye spraying

a

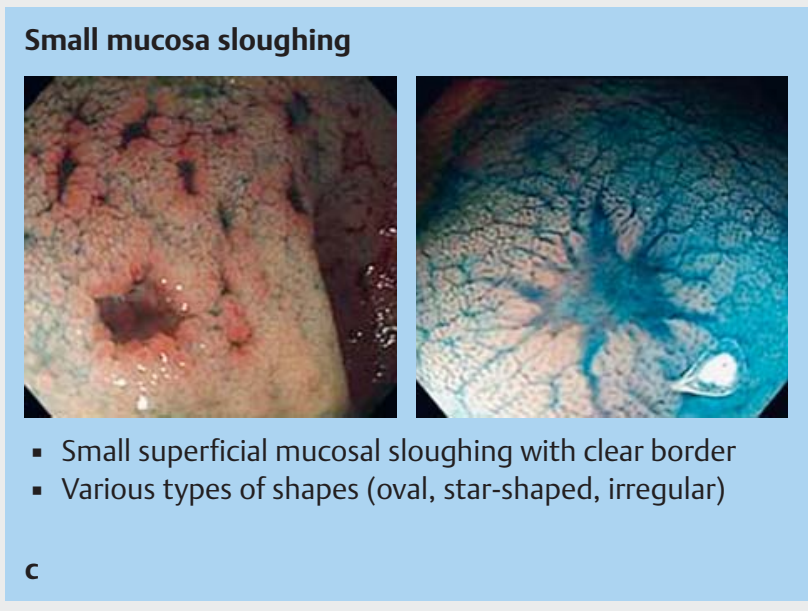

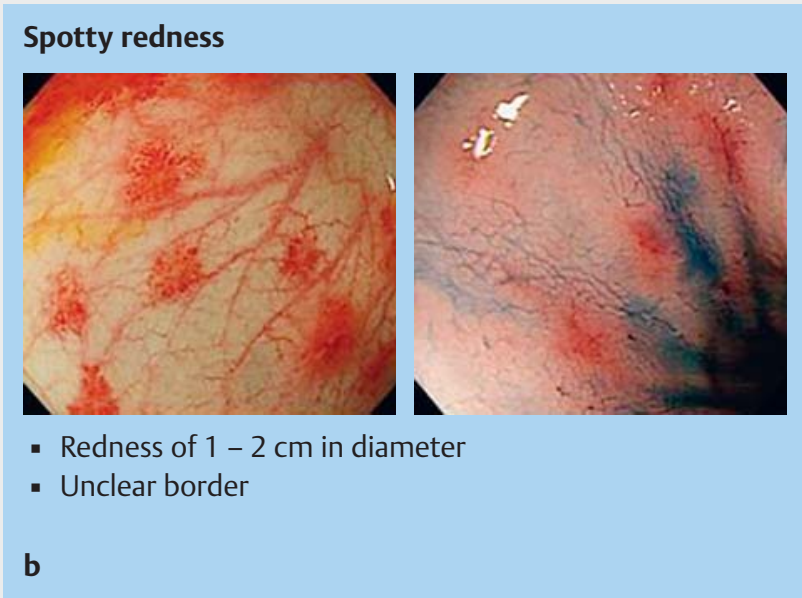

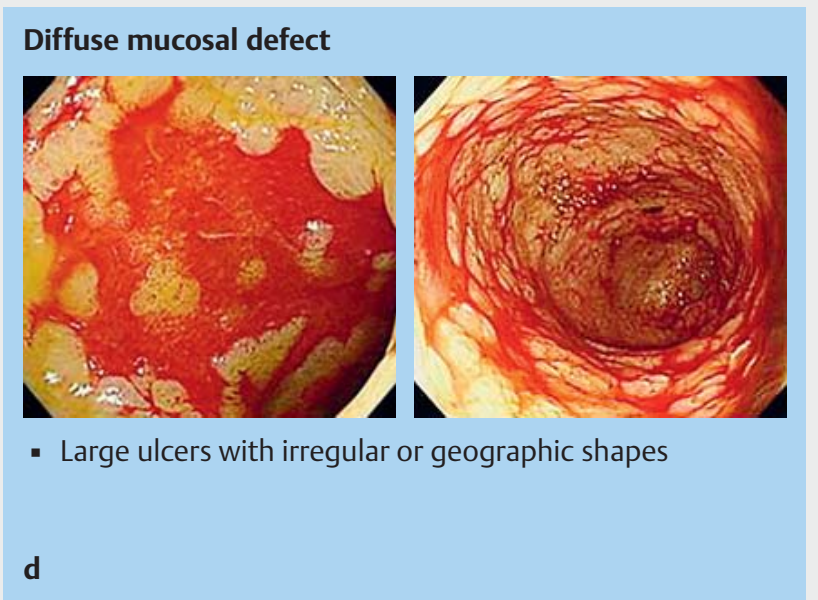

- Fig. 1 Definitions of the 4 characteristic findings. a Orange peel appearance: mucosal edema with granular surface looking like "orange peel" or "tortoiseshell," which was usually highlighted with indigo carmine dye spraying. b Spotty redness: redness of 1 to $2 \mathrm{~cm}$ in diameter with an unclear border. c Small mucosal sloughing: a small superficial area of mucosal sloughing with various types of shapes, with a clear border. $\mathbf{d}$ Diffuse mucosal defects: large ulcers with irregular or geographic shapes.

\section{Evaluation of the relation between biopsy sites and the histological findings}

The relationship between the colonoscopic features of the biopsy sites and the corresponding histological findings were evaluated based on a case-by-case review. The percentage of the pathological crypt apoptosis in the biopsy specimens was calculated for each endoscopic feature, respectively.

\section{Statistics}

The comparison of the positive rates of each endoscopic finding between transplantation types was analyzed by Chi-squared test, and a $P<0.05$ was considered statistically significant. All statistics analyses were performed using JMP Pro 11.0.0. (SAS Institute Inc., USA).

\section{Results}

\section{Patients' background}

Between January 2001 and December 2015, 93 patients with suspicious symptoms of gastrointestinal GVHD after allogenic HSCT received colonoscopic evaluations at Tohoku University Hospital. A flow diagram of the cases is presented in $\mathbf{r i g .} \mathbf{2}$. Of the 93 patients, 72 were diagnosed with gastrointestinal GVHD, based on the histological findings. Two of the 72 cases were complicated with cytomegalovirus (CMV) colitis, proven via CMV immunohistochemistry. Seventy patients with histologically proven gastrointestinal GVHD, without CMV infection, were enrolled in this study.

Baseline characteristics of the patients are summarized in - Table 1. Patients included 33 males and 37 females (median age 39 years; range $2-63$ years). Median time of colonoscopy after transplant was 42 days (range 15-159 days). The underlying diseases were acute leukemia $(n=45)$, chronic leukemia 


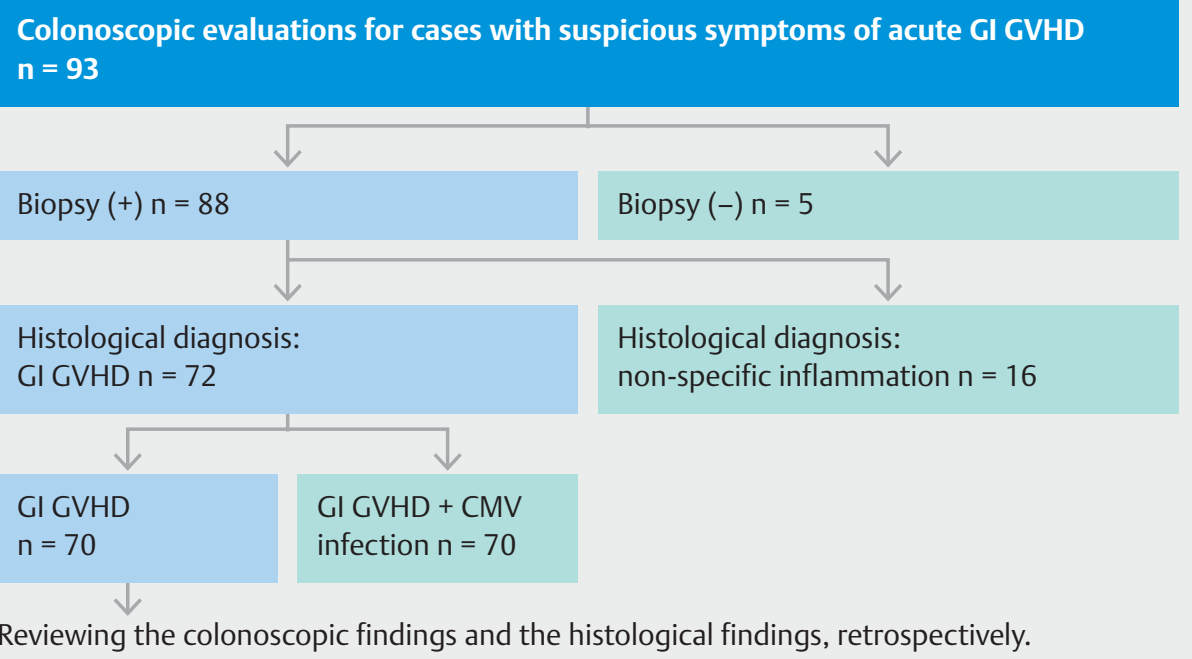

- Fig. 2 Flow diagram of the cases. A total of 93 patients with suspicious symptoms of gastrointestinal GVHD after allogenic HSCT received colonoscopic evaluations. Out of all 93 patients, 72 were pathologically diagnosed with gastrointestinal GVHD. Seventy cases with histologically proven gastrointestinal GVHD without CMV infection were finally enrolled in this study. GI GVHD, gastrointestinal graft-vs-host disease; $\mathrm{HSCT}$, hematopoietic stem cell transplantation; CMV, cytomegalovirus

- Table 1 Baseline characteristics of patients.

Sex male/ female

$33 / 37$

Age at colonoscopy (days ${ }^{1}$ )

$39(2-63)$

Days of colonoscopy after transplant (days ${ }^{1}$ )

$42(15-159)$

Underlying Ddagnosis

- AML (acute myelocytic leukemia)

31

- ALL (acute lymphocytic leukemia)

14

- CML (chronic myelocytic leukemia)

- ML (malignant lymphoma)

9

- ATL (adult T-cell lymphoma)

3

- MDS (myelodysplastic syndrome)

10

- Others

2

Type of transplant

- BMT (bone marrow transplantation)

31

- PBCS (peripheral blood stem cell transplantation)

- CBT (cord blood transplantation)

Data were shown as median and range

$(n=1)$, malignant lymphoma $(n=9)$, adult T-cell leukemia $(n=$ $3)$, myelodysplastic syndrome $(n=10)$, and others $(n=2)$. The types of transplantation were bone marrow transplantation ( $\mathrm{n}$ $=31$ ), peripheral blood stem cell transplantation $(n=11)$, and cord blood transplantation $(\mathrm{n}=28)$.

\section{Percentage of the 4 characteristic findings in 70 cases with gastrointestinal GVHD}

Orange peel appearance was observed in 66 of 70 cases (94.3\%). Spotty redness was observed in 45 of 70 cases (64.3\%). Small mucosal sloughing was observed in 49 of 70 cases $(70.0 \%)$. Diffuse mucosal defects were observed in six of 70 cases $(8.6 \%)$. In the positive case with each finding, the finding was detected as in a continuous pattern. Pictures of representative cases with each finding are shown in > Fig. 3, > Fig. 4, - Fig.5, Fig.6. The sample pictures in each figure were selected from independent cases. There was no association between the endoscopic findings and the transplantation types ( Table 2).

Combinations of the 4 characteristic colonoscopic findings are shown in $\mathbf{F i g . 7}$. There was only 1 patient who did not have any of the 4 findings. Four of 70 cases (5.2\%) presented with all 4 findings. The number of cases that were concurrently positive for 1, 2, and 3 findings was 16 (20.8\%), 20 (26.0\%), and 34 (48.6\%), respectively.

The final scoping portion and the numbers of cases are shown in > Fig. 8. Fifty-eight of 70 cases $(82.9 \%)$ were investigated using rectosigmoidoscopy. All of those cases presented with at least 1 of the 4 characteristic findings in the rectosigmoid portion. There were 12 patients $(17.1 \%)$ in whom colonoscopic observations were performed beyond the sigmoid colon; total colonoscopies were performed in only 4 cases. All of these 12 cases presented with at least 1 of the 4 characteristic findings up to the sigmoid colon. Overall, all of the 70 cases showed at least 1 of the 4 characteristic findings in the rectosigmoid portion. 


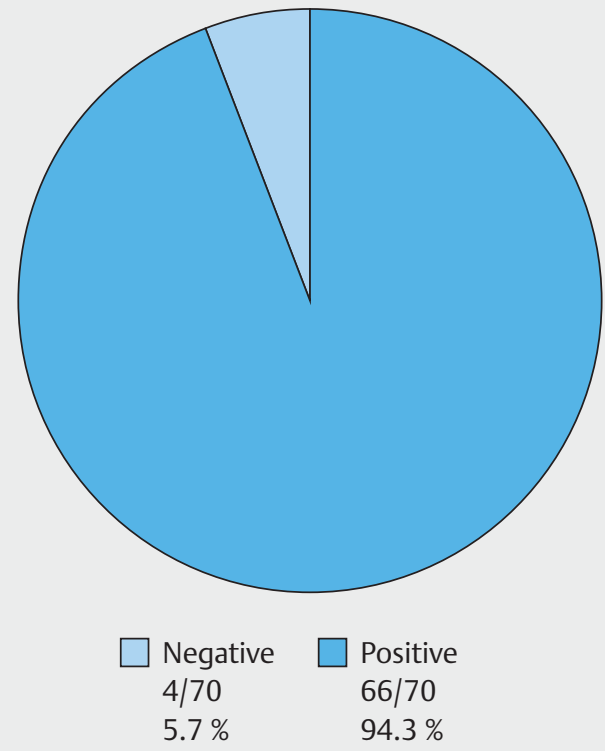

Examples of the positive cases

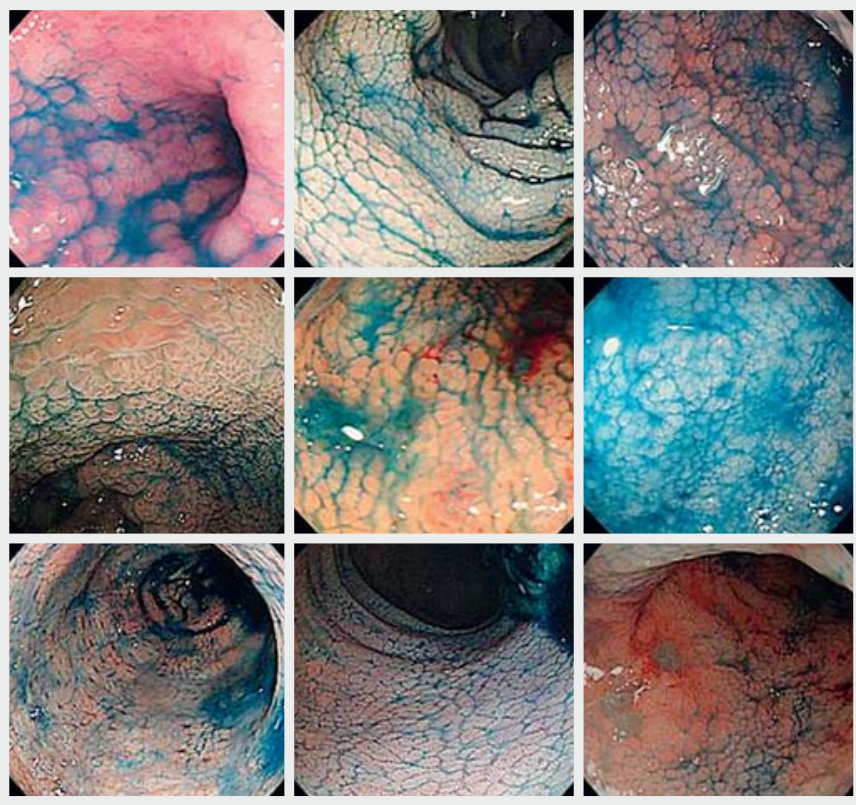

- Fig. 3 Percentage and examples of the positive cases with orange peel appearance. Orange peel appearance was observed in 66 of 70 cases (94.3\%).

\section{Spotty redness}

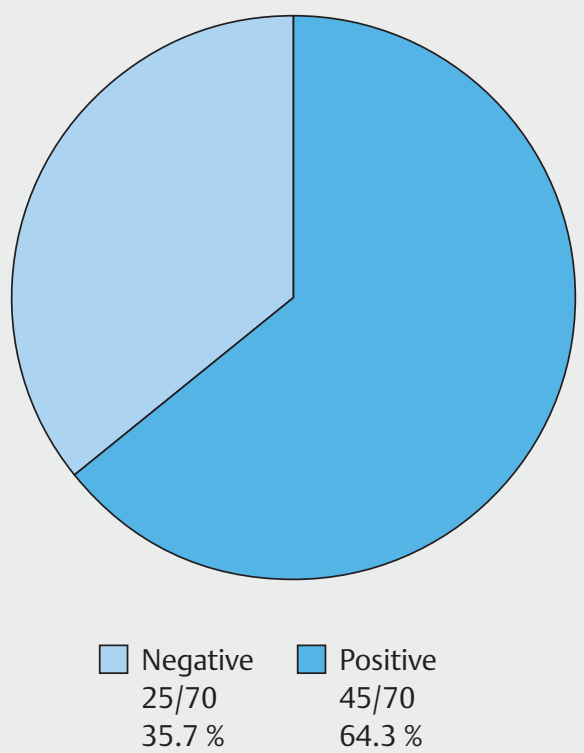

Examples of the positive cases

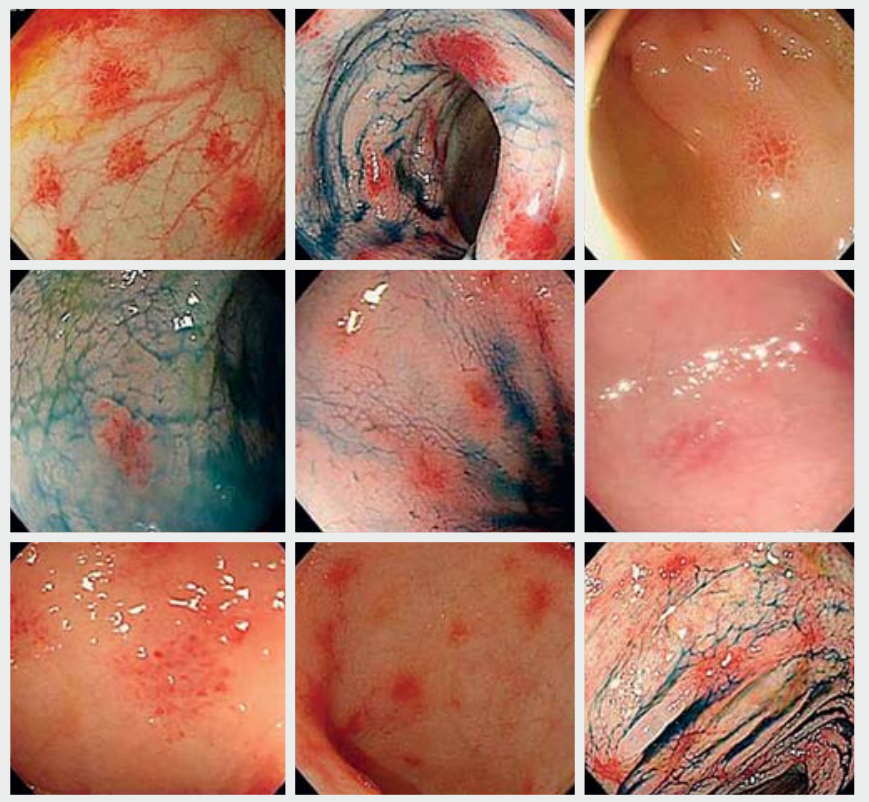

Dig. 4 Percentage and examples of the positive cases with spotty redness. Spotty redness was observed in 45 of 70 cases (64.3\%). 


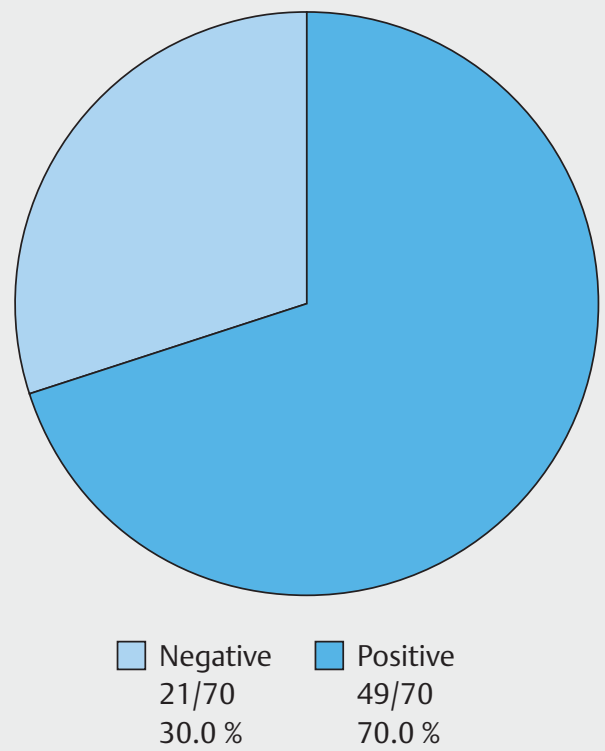

Examples of the positive cases
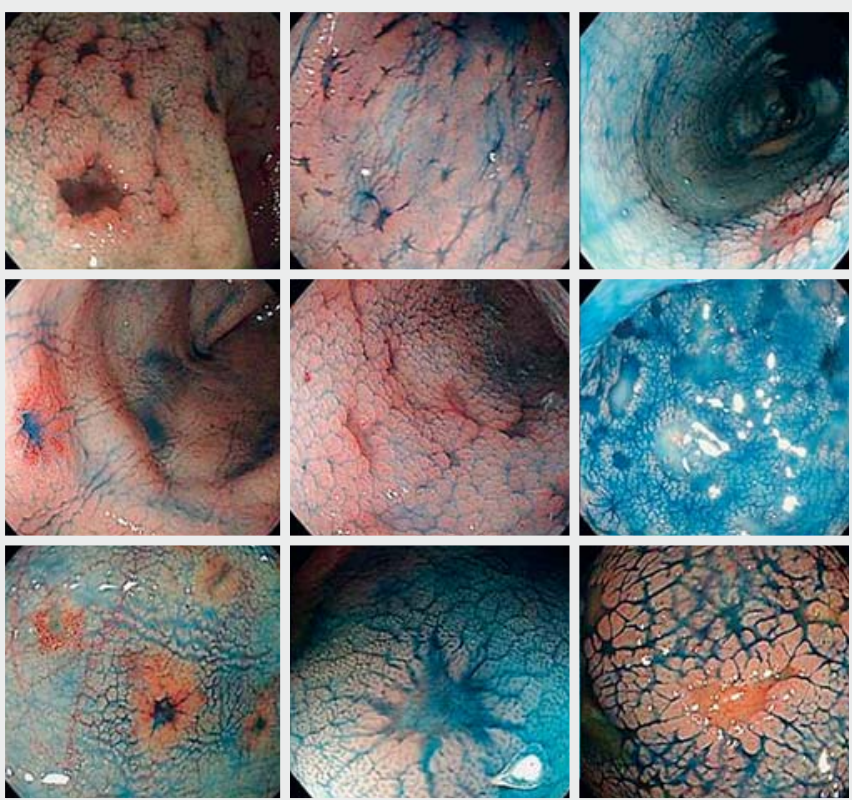

- Fig. 5 Percentage and examples of the positive cases with small mucosal sloughing. Small mucosal sloughing was observed in 49 of 70 cases (70.0\%).

\section{Diffuse mucosal effect}

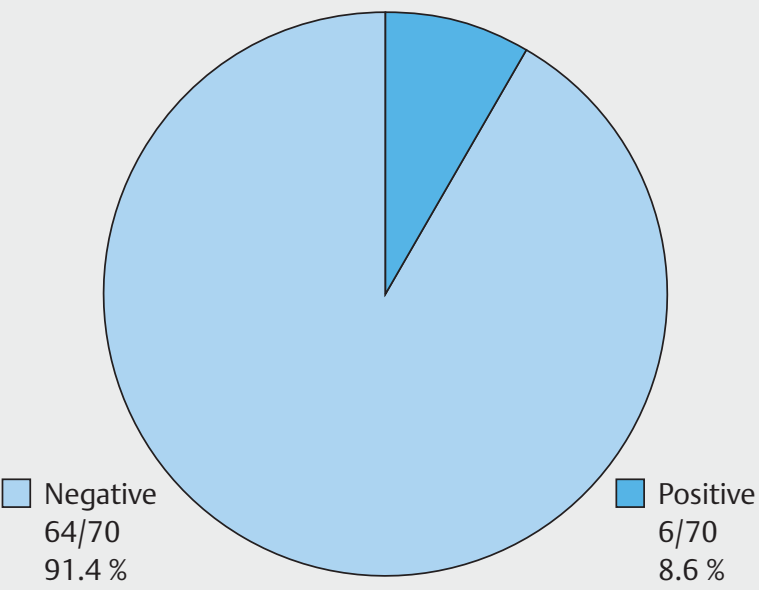

Examples of the positive cases
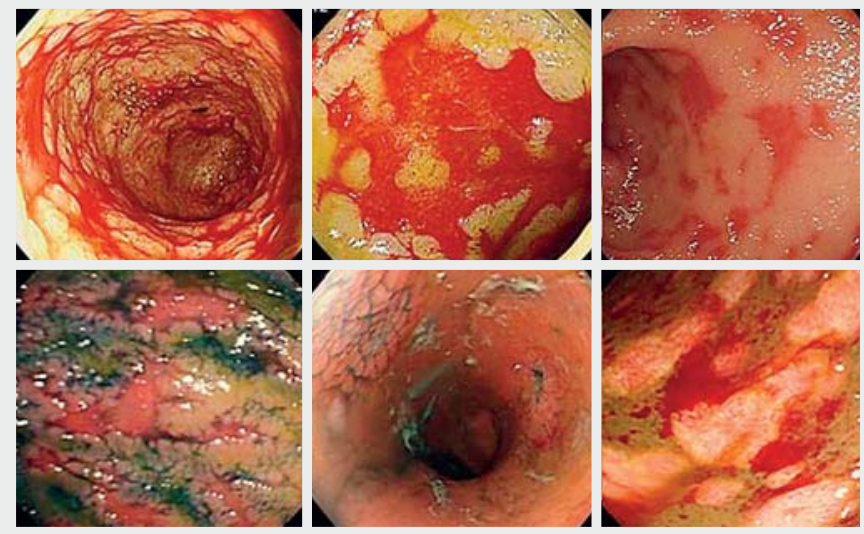

Fig. 6 Percentage and examples of the positive cases with diffuse mucosal defect. Diffuse mucosal defect was observed in 6 of 70 cases (8.6\%).

\section{Percentage of crypt apoptosis in biopsy specimens taken from the 4 characteristic findings}

A total of 197 biopsies were performed in 70 patients. The corresponding endoscopic features of the biopsy sites could be identified in 156 out of 197 biopsies by reviewing the endos- copy reports. The relationship between the colonoscopic features of the 156 biopsy sites and the corresponding histological findings were evaluated based on a case-by-case review. Total biopsy numbers and the percentage of detection of the histological crypt apoptosis from each colonoscopic feature are summarized in $>$ Table 3 . The percentages of crypt apoptosis in the 
- Table 2 Comparisons of positive rates for each endoscopic finding between the transplantation types.

\begin{tabular}{|l|l|l|l|l|}
\hline & $\begin{array}{l}\text { BMT } \\
\mathbf{n}=\mathbf{3 1}\end{array}$ & $\begin{array}{l}\text { PBSCT } \\
\mathbf{n = 1 1}\end{array}$ & $\begin{array}{l}\text { CBT } \\
\mathbf{n = 2 8}\end{array}$ \\
\hline Orange peel appearance & $29(93.5 \%)$ & $11(100 \%)$ & $26(92.9 \%)$ & 0.80 \\
\hline Spotty redness & $22(71.0 \%)$ & $5(45.5 \%)$ & $18(64.3 \%)$ & 2.32 \\
\hline Small mucosal sloughing & $23(74.2 \%)$ & $9(81.8 \%)$ & $17(60.7 \%)$ & 2.14 \\
\hline Diffuse mucosal defect & $4(12.9 \%)$ & $1(9.1 \%)$ & $1(3.6 \%)$ & 1.60 \\
\hline $\begin{array}{l}\text { BMT, bone marrow transplantation; } \\
\text { 1 Chi-squared test }\end{array}$ & & & \\
\hline
\end{tabular}

Combinations of the four characteristic findings

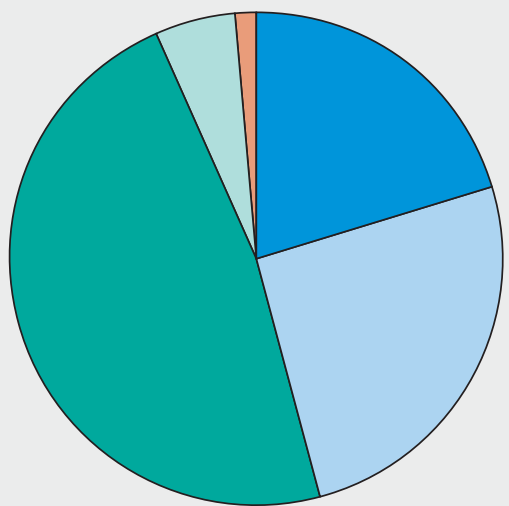

$\square$ Positive for one finding $16 / 70$ (20.8\%)

Positive for four findings 4/70

(5.2\%) $\square$ Positive for two findings 20/70 (26.0\%)

Positive for three findings 34/70 (48.6\%)

Negative for all four findings $1 / 70$ (1.4\%)
- Fig. 7 Combinations of the 4 characteristic findings. There was only 1 patient who did not have any of the 4 findings. Four of 70 patients (5.2\%) presented with all 4 findings. The number of patients who were concurrently positive for 1,2 , and 3 findings were $16(20.8 \%), 20$ (26.0\%), and 34 (48.6\%), respectively.

biopsy specimens from orange peel appearance, spotty redness, small mucosal sloughing, and diffuse mucosal defect were $87.5 \%, 83.3 \%, 87.2 \%$, and $88.9 \%$, respectively. In the specimens taken from endoscopically normal mucosa, crypt apoptosis was detected in 7 of 8 biopsies.

Colonoscopic findings and corresponding histological findings of a typical case, which presented with a combination of 2 characteristic findings (orange peel appearance and small mucosal sloughing), are shown in $>$ Fig.9. Crypt apoptosis was found in the biopsy from the area with the orange peel appear- ance, and destruction of goblet cells was found in the biopsy from small mucosal sloughing.

\section{Discussion}

In the current study, we evaluated the usefulness of the four characteristic colonoscopic findings for diagnosing gastrointestinal GVHD. In our samples, orange peel appearance was observed in more than $90 \%$ of the gastrointestinal GVHD patients. Indigo carmine dye spray facilitated identification of this finding. In a few cases, it was not difficult to detect orange peel appearance only by the white-light. However, in most of the cases, indigo carmine was necessary and helped us easier to identify orange peel appearance. Since, we argue that the chromoendscopy with indigo carmine dye spraying should be used in the suspected case with gastrointestinal GVHD. The previous study also reported that the orange peel appearance was a characteristic finding of gastrointestinal GVHD [15-18]. The orange peel appearance reflects the overdilatation of the superficial innominate groove of the colonic mucosa, induced by submucosal edema. We identified high rates $(87.5 \%)$ of gland apoptosis in the biopsies taken from the areas with an orange peel appearance. Therefore, identifying the orange peel appearance, using indigo carmine dye spray, and taking a biopsy from this area could be necessary to diagnose gastrointestinal GVHD efficiently.

Spotty redness has been reported as a useful finding to diagnose gastrointestinal GVHD [17]. However, the sample size in the previous study was too small to establish reliable evidence. Our study, including 70 cases of gastrointestinal GVHD, identified more than $60 \%$ positive cases of spotty redness. By reviewing the colonoscopic features and the histological findings case-by-case, biopsy specimens of spotty redness showed that the lymphocytes' invasions into the crypt had high rates of crypt cell apoptosis (83.3\%). We consider that the spotty redness could reflect the focal inflammation of a single or several of the crypts, induced by GVHD.

Small mucosal sloughing was also reported as a characteristic finding of gastrointestinal GVHD $[17,18]$. In the current study, $70 \%$ of cases were positive for this finding. As presented in $>$ Fig. 5, the positive cases for this finding showed multiple areas of small superficial mucosal sloughing with clear borders. The shapes of the areas of mucosal sloughing varied among the 


\section{The final scoping proportions and the number of the cases}

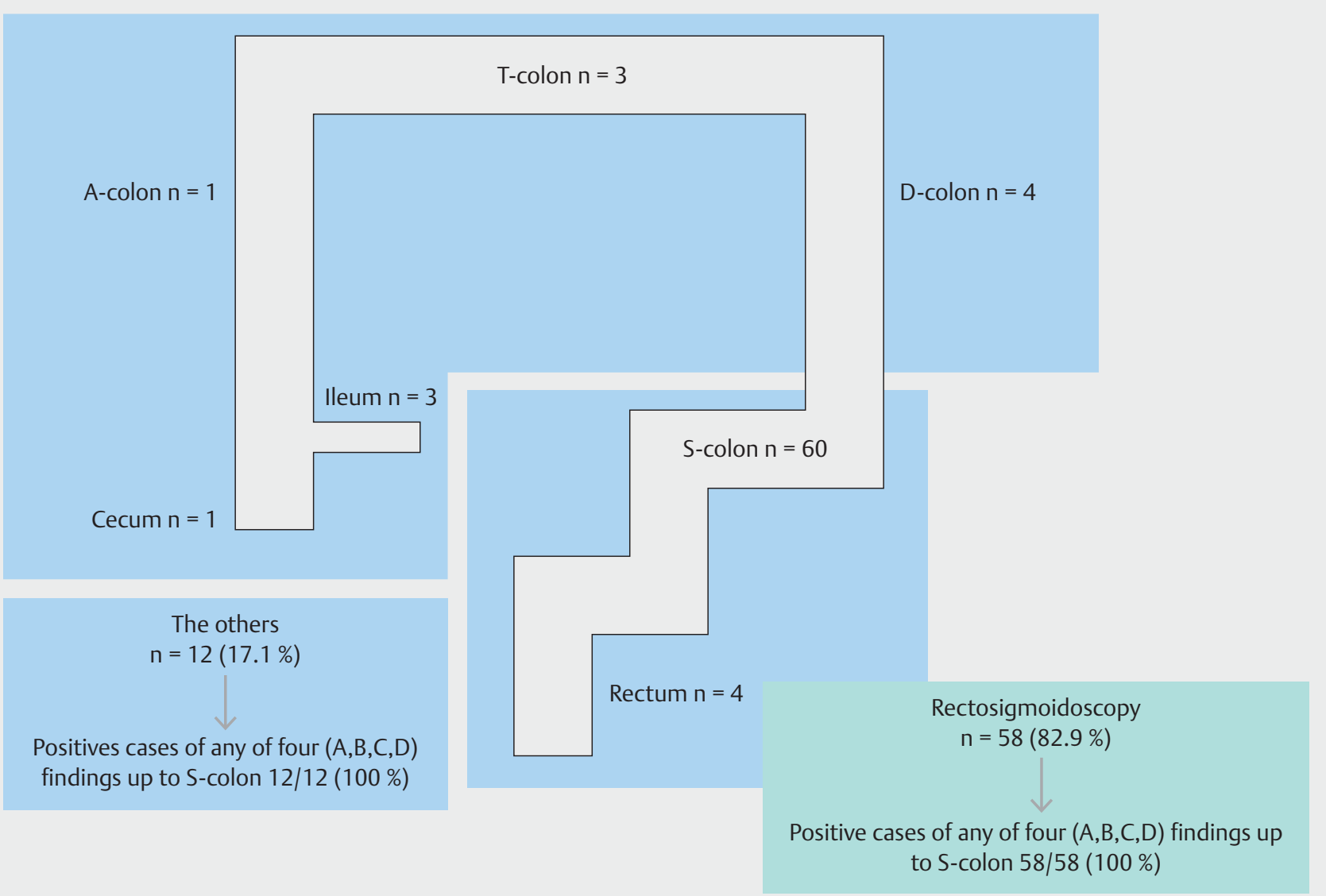

- Fig. 8 The final scoping portion and the number of the cases. Fifty-eight of 70 patients ( $82.9 \%)$ were investigated with rectosigmoidoscopy. All of these 58 cases presented with at least 1 of the 4 characteristic findings in the rectosigmoid portion. There were 12 patients (17.1\%) in whom colonoscopic observations were performed beyond the sigmoid colon. All of these 12 cases presented with at least 1 of the 4 characteristic findings up to the sigmoid colon.

- Table 3 Percentage of histological crypt apoptosis in the biopsy specimens from each endoscopic feature.

\begin{tabular}{|l|c|c|c|}
\hline Endoscopic features & Total biopsy number (n) & Positive for crypt apoptosis (n) & $\begin{array}{l}\text { Percentage of detection for } \\
\text { crypt apoptosis (\%) }\end{array}$ \\
\hline Orange peel appearance & 48 & 42 & 87.5 \\
\hline Spotty redness & 36 & 30 & 83.3 \\
\hline Small mucosal sloughing & 55 & 48 & 87.2 \\
\hline Diffuse mucosal defect & 9 & 8 & 88.9 \\
\hline Endoscopically normal mucosa & 8 & 7 & 87.5 \\
\hline Total & 156 & 135 & 86.5 \\
\hline
\end{tabular}

cases, such as round, star-like, or irregular. Biopsy specimens from these areas revealed a high rate of crypt apoptosis (87.2\%). As shown in > Fig. 9, the histological findings in the areas of small mucosal sloughing tended to be more aggressive than those in the areas with orange peel appearance and spotty redness, in which the crypt destruction or gland necrosis were more frequently observed. We postulate that adhesions of several areas of gland necrosis could gradually become the area of focal small mucosal sloughing.

Although diffuse mucosal defects were observed in only $8.6 \%$ of our cases, this finding tended to be observed in the cases with severe gastrointestinal GVHD. We hypothesize that 
Case: 51 y. o. female, intestinal GVHD, day 42 after CBT for AML
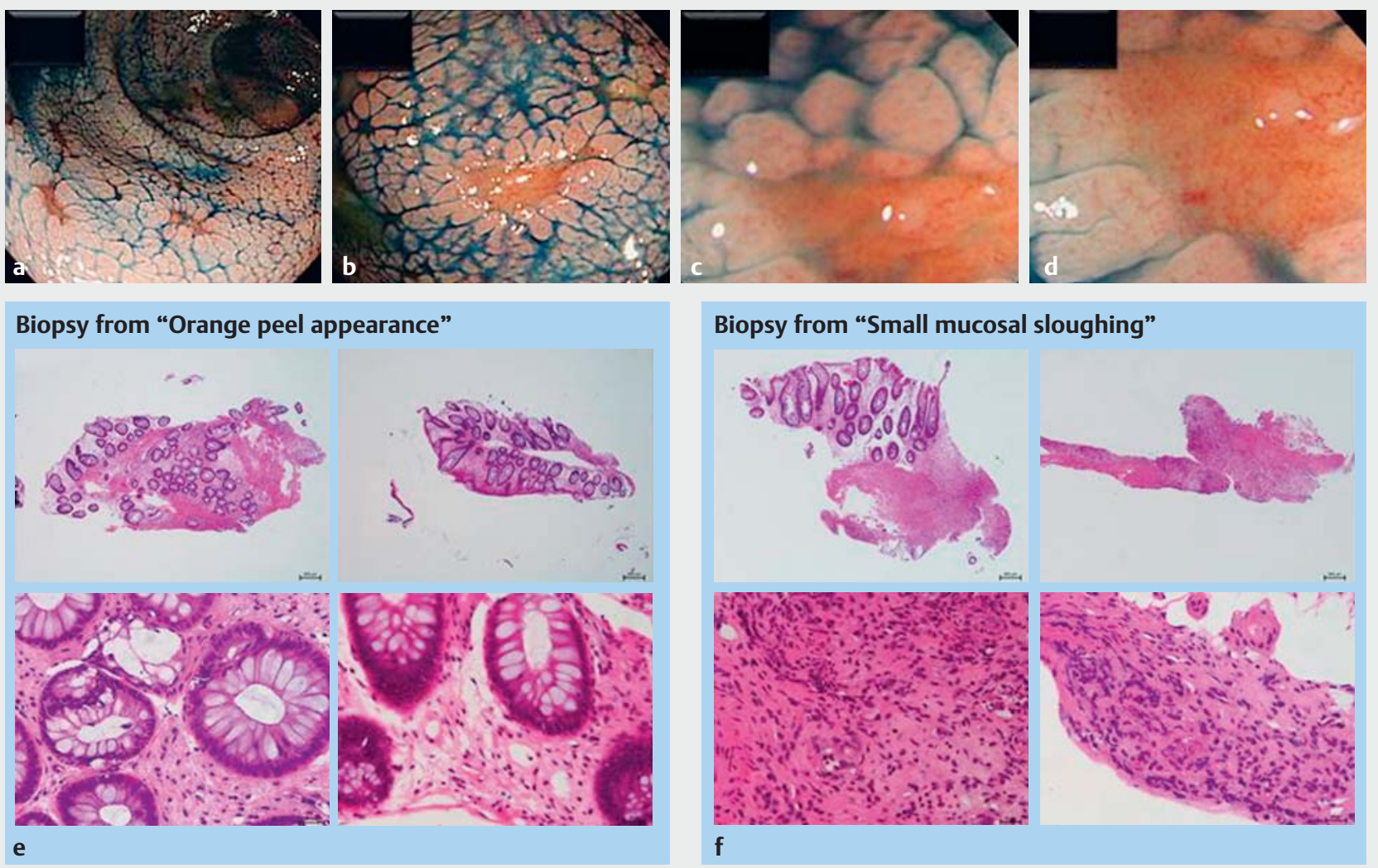

- Fig. 9 Colonoscopic and histological findings of a typical case with orange peel appearance and small mucosal sloughing. The patient was a 51-year-old female with gastrointestinal GVHD. The colonoscopic and histological examinations were performed at Day 42 after CBT for AML. a, b Orange peel appearance and small mucosal sloughing were observed. c, $\mathbf{d}$ Magnified endoscopic findings of the small mucosal sloughing. e Histological findings of the biopsy specimen taken from an area with an orange peel appearance. Crypt apoptosis was found. $\mathbf{f}$ Histological findings of the biopsy specimen taken from an area of small mucosal sloughing. Destruction of goblet cells was found.

gastrointestinal GVHD, gastrointestinal graft-vs-host disease; CBT, cord blood transplantation; AML, acute myeloid leukemia.

diffuse and severe crypt necrosis lead to the enlarged mucosal defects.

Reviewing our cases, we hypothesize that the 4 characteristic findings discovered in the current study could appear gradually and successively as the gastrointestinal GVHD proceeds ( $\vee$ Fig. 10). There are 2 major reasons for this hypothesis. The first reason is that many of our cases were positive for 2 or more findings. Altogether, approximately $80 \%$ of our cases were positive for 2 or more findings, and more than $50 \%$ of the cases were positive for 3 or more findings. The second reason is that there were several cases with intermediate findings, in which it was difficult to distinguish spotty redness from small mucosal sloughing, or small mucosal sloughing from diffuse mucosal defects. These reasons could support the hypothesis that gastrointestinal GVHD shows the sequential transition of the characteristic colonoscopic findings. Therefore, it could be quite important to identify the four characteristic findings and their combinations to diagnose gastrointestinal GVHD based on the colonoscopic findings.
There are still some issues concerning the endoscopic diagnosing strategy for gastrointestinal GVHD. First, it is still controversial whether scoping up to the cecum or terminal ileum is necessary or not, when performing a colonoscopy for a suspicious case of gastrointestinal GVHD. In the past, there was a report describing the usefulness of magnifying endoscopy for the terminal ileum [19]. However, recent studies indicated that the rectosigmoidoscopy is sufficient for diagnosing gastrointestinal GVHD $[8,10-12]$. It could be ideal to scope up to the proximal colon or the ileum to identify the activity of GVHD more precisely. However, the patients with gastrointestinal GVHD after HSCT are usually in poor general condition, with hematopenia, dehydration, and electrolyte imbalance due to severe diarrhea, and are immunosuppressed. Performing total colonoscopy might be too invasive and iatrogenic bowel perforation could be fatal for such patients. Furthermore, our clinical experiences indicate that total colonoscopy can be difficult for the patient with gastrointestinal GVHD because of severe abdominal pain. Our current study showed that all of the cases showed at least one of the four characteristic findings in the rectosigmoid por- 


\section{Hypothesis: sequential transition of colonoscopic findings}

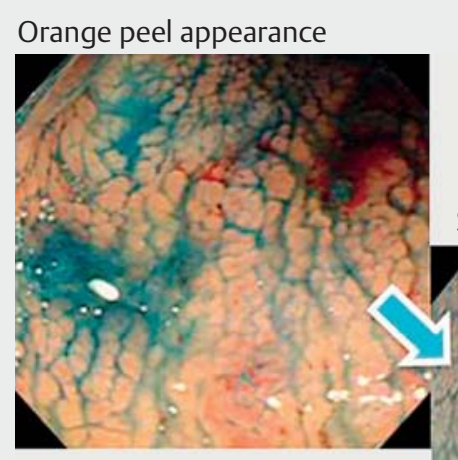

Histology:

Submucosal edema

Spotty redness

Histology:

Lymphocyte infiltration

Crypt cell apoptosis

Small mucosal sloughing

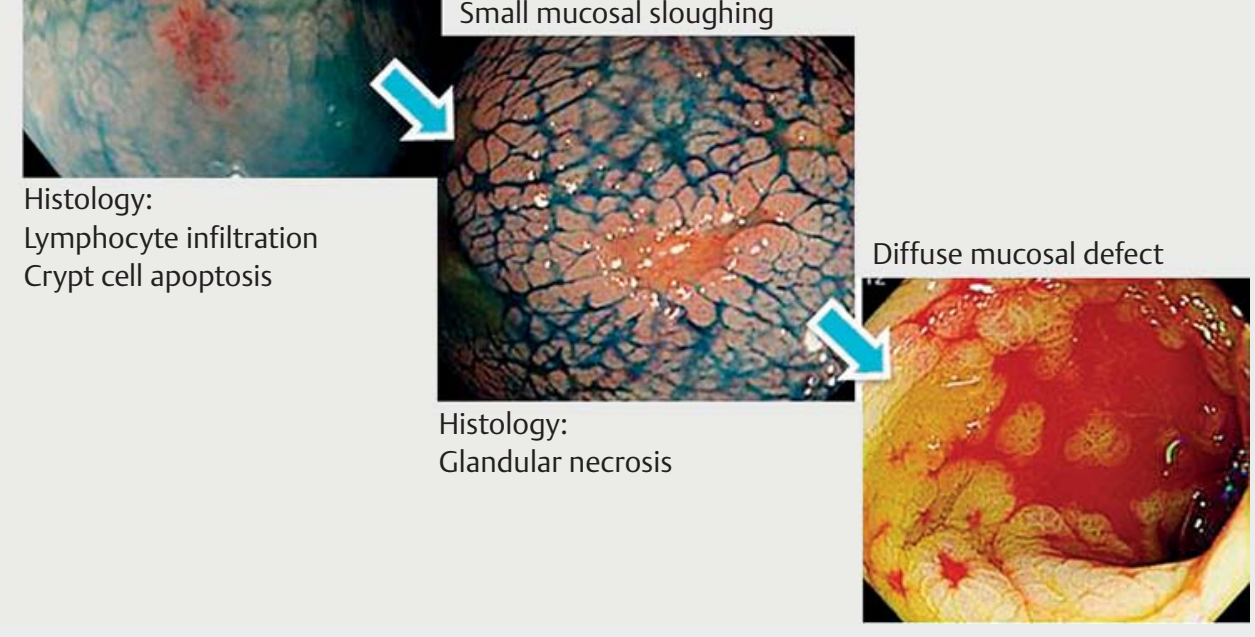

Fig. 10 Hypothesis: Sequential transition of colonoscopic findings. The 4 characteristic findings picked up in the current study could appear gradually and successively as the gastrointestinal GVHD proceeds.

tion. The detection rate of crypt cell apoptosis with at least 1 of the 4 findings was more than $80 \%$. Furthermore, all of the cases were diagnosed as GVHD based on the biopsy from the rectosigmoid portion (data was not shown). Second, it would be also controversial whether magnifying procedure is useful for diagnosing gastrointestinal GVHD. Based on our experiences, magnifying scope would not be necessary for detecting the four characteristic findings studied in this study. We think that further experiences or the prospective study should be needed for this issue. Finally, biopsy site is another important issue. It is still unclear whether the random biopsy with no precise endoscopic observation is enough to diagnose gastrointestinal GVHD. In the present study, 148 out of 156 biopsies were taken from any of the four findings, and only 8 biopsies were taken from the endscopically normal mucosa. Our results showed the high detection rates for crypt apoptosis. Based on these results, we think that target biopsy would be the best strategy for efficient and less invasive diagnostic strategy for gastrointestinal GVHD.

From the previous evidence and these results, we propose an efficient and less invasive diagnostic strategy for gastrointestinal GVHD. For the suspicious cases of gastrointestinal GVHD, rectosigmoidoscopy could be the first procedure performed.
Identifying the 4 characteristic findings on rectosigmoidoscopy and taking biopsies from the areas with these findings is essential. Indigo carmine dye spraying is strongly recommended to detect the orange peel appearance. Total colonoscopy should be performed only when the rectosigmoid portion reveals normal mucosal findings. Even if the colonoscopic findings are completely normal, a biopsy from the endoscopically normal mucosa should be performed, because it is helpful in some cases.

There are some important limitations in the current study. The first is that we could not include the negative controls, which were not diagnosed as gastrointestinal GVHD. There were a few cases, in which colonoscopy was performed and the patient was not diagnosed as gastrointestinal GVHD after HSCT. Therefore, we could only calculate the sensitivity of the 4 characteristic findings for diagnosing gastrointestinal GVHD, but not the specificity. The second is that the validations of endoscopic findings between individual endoscopists were not performed because of the retrospective study design. The third is that the correlation between colonoscopic findings and the treatment responses or the prognosis were not evaluated. The further study should be needed to clarify this issue. 


\section{Conclusion}

In conclusion, orange peel appearance, spotty redness, small mucosal sloughing, and diffuse mucosal defect are the characteristic colonoscopic findings that are useful for diagnosis of gastrointestinal GVHD. These findings are frequently observed in the rectosigmoid portion, often with combinations of these findings. The detection rates for crypt cell apoptosis in the biopsy specimens from these findings are quite high. Identifying the four characteristic findings on rectosigmoidoscopy and taking biopsies from these findings is essential for the efficient and less invasive strategy for diagnosing gastrointestinal GVHD.

\section{Competing interests}

None

\section{References}

[1] Gratwohl A, Brand R, Apperley J et al. Graft-versus-host disease and outcome in HLA-identical sibling transplantations for chronic myeloid leukemia. Blood 2002; 100: 3877-3886

[2] Przepiorka D, Weisdorf D, Martin P et al. 1994 Consensus Conference on Acute GVHD Grading. Bone Marrow Transplant 1995; 15: 825 828

[3] Nomura K, lizuka T, Kaji D et al. Clinicopathological features of patients with acute graft-versus-host disease of the upper digestive tract. J Gastroenterol Hepatol 2014; 29: 1867 - 1872

[4] Ross WA, Ghosh S, Dekovich AA et al. Endoscopic biopsy diagnosis of acute gastrointestinal graft-versus-host disease: rectosigmoid biopsies are more sensitive than upper gastrointestinal biopsies. Am J Gastroenterol 2008; 103: 982-989

[5] Sultan M, Ramprasad J, Jensen MK et al. Endoscopic diagnosis of pediatric acute gastrointestinal graft-versus-host disease. J Ped Gastroenterol Nutr 2012; 55: 417-420

[6] Crowell KR, Patel RA, Fluchel M et al. Endoscopy in the diagnosis of intestinal graft-versus-host disease: is lower endoscopy with biopsy as effective in diagnosis as upper endoscopy combined with lower endoscopy? Ped Blood Cancer 2013; 60: 1798 - 1800
[7] Lee KJ, Choi S], Yang HR et al. Stepwise endoscopy based on sigmoidoscopy in evaluating pediatric graft-versus-host disease. Ped Gastroenterol Hepatol Nutr 2016; 19: 29-37

[8] Ma C, Maluf HM, Liu TC. Acute graft-versus-host disease is more prevalent and severe in the lower than the upper gastrointestinal tract. Human Pathology 2015; 46: 1480-1487

[9] Wild D, Sung AD, Cardona D et al. The diagnostic yield of site and symptom-based biopsies for acute gastrointestinal graft-versus-host disease: A 5-year retrospective review. Dig Dis Sci 2016; 61: 806 - 813

[10] Johansson JE, Nilsson O, Stotzer PO. Colonoscopy and sigmoidoscopy are equally effective for the diagnosis of colonic acute graft-versushost disease in patients with diarrhea after allogeneic stem cell transplantation: A prospective controlled trial. Biol Blood Marrow Transplantation 2015; 21: 2086-2090

[11] Ip S, Marquez V, Schaeffer DF et al. Sensitivities of biopsy sites in the endoscopic evaluation of graft-versus-host disease: Retrospective review from a tertiary center. Dig Dis Sci 2016: doi:10.1007/s10620$016-4142-1$

[12] Minamino $\mathrm{H}$, Machida $\mathrm{H}$, Tominaga $\mathrm{K}$ et al. Rectal biopsy, rather than ileal, is appropriate to confirm the diagnosis of early gastrointestinal graft-versus-host disease. Scand J Gastroenterol 2015; 50: 1428 1434

[13] Kreisel W, Dahlberg M, Bertz H et al. Endoscopic diagnosis of acute intestinal GVHD following allogeneic hematopoietic SCT: a retrospective analysis in 175 patients. Bone Marrow Transplantation 2012; 47: $430-438$

[14] Roy J, Snover D, Weisdorf S et al. Simultaneous upper and lower endoscopic biopsy in the diagnosis of intestinal graft-versus-host disease. Transplantation 1991; 51: 642-646

[15] Mashiba T, Usami A, Soga Y et al. Endoscopic findings of intestinal graft-versus-host disease following allogenic bone marrow and peripheral blood stem cell transplantation. [In Japanese with English abstract]. Gastroenterol Endosc 2003; 45: 929-934

[16] Ponec RJ, Hackman RC, McDonald GB. Endoscopic and histologic diagnosis of intestinal graft-versus-host disease after marrow transplantation. Gastrointest Endoscop 1999; 49: 612-621

[17] Oomori S, Takagi S, Kikuchi T et al. Significance of colonoscopy in patients with intestinal graft-versus-host disease after hematopoietic stem cell transplantation. Endoscop 2005; 37: 346 - 350

[18] Hiejima E, Nakase H, Matsuura M et al. Diagnostic accuracy of endoscopic features of pediatric acute gastrointestinal graft-versus-host disease. Dig Endoscop 2016: doi:10.1111/den.12604

[19] Onozawa M, Yonezumi M, Kawarazaki M et al. Usefulness of magnifying endoscopic evaluation of the terminal ileum for a patient with graft-versus-host disease after allogeneic hematopoietic stem cell transplantation. Ann Hhematol 2005; 84: $106-109$ 